\title{
A clinical analysis of an intrahepatic lesion detected in a 65-year-old lady who admitted for severe epigastric pain
}

\author{
Massimo Bolognesi* \\ Internal General Medicine, Department of General Practice Medicine, AUSL della Romagna - District of Cesena, Italy
}

\begin{abstract}
Although there are a number of published case reports of hepatic subcapsular fluid collection, it is not straightforward to make a correct diagnosis when both imaging and laboratory test are equivocal and unclear for physicians. Lack of experience and specific literature makes diagnosis and management of intrahepatic lesions or fluid collection very difficult. Here the author just wants to describe the clinical presentation and outcome in a 65 -year-old lady with severe onset of epigastric pain in absence of fever but with imaging of the intrahepatic subcapsular lesion in the left lobe.
\end{abstract}

\section{Introduction}

The evaluation of abdominal pain requires an understanding of the possible mechanisms responsible for pain, a broad differential of common causes, and recognition of typical patterns and clinical presentations. Focal lesions of the liver in the adult can be classified as developmental, neoplastic, inflammatory, or miscellaneous lesions. Many overlapping characteristics of these lesions frequently lead to a long list of differential diagnoses with a subsequent lengthy and expensive work-up for the patient. Unfortunately, in very few cases, familiarity with the most relevant radiological key features, in combination with little critical clinical information, cannot provide sufficient information for the characterization. This article presents the clinical scenario of an afebrile, smoker and hypertensive nondiabetic 65-year-old lady who goes to her GP's surgery complaining of a sudden epigastric pain radiating to the left shoulder suggestive for acute pancreatitis, then admitted to hospital for further investigations and discharged with diagnosis of sepsis caused by hepatic abscess.

\section{Case report}

A smoker, hypertensive non-diabetic 65-year-old woman presented to her GP's office with severe epigastric pain radiating to the left shoulder, which had started the previous day. Her blood pressure was $140 / 80 \mathrm{~mm} \mathrm{Hg}$, pulse 76 beats per minute, and the ECG was completely normal. Physical examination revealed upper abdominal tenderness with a severe pain at palpation in the epigastric region. Abdominal perforation and intestinal occlusion were ruled out quickly. She did not have significant weight loss, gastrointestinal bleeding, pedal edema, jaundice, and no fever. Medical and family history were unremarkable for biliary or hepato- pancreatic disease. Recently she had had corticosteroid therapy for idiopathic urticaria and angioedema for 10 days. After a general examination the patient was soon transferred to emergency with diagnosis of suspected acute pancreatitis. The laboratory parameters showed a total leucocyte count of $26200 / \mathrm{mm}^{3}$ with a neutrophilic predominance, mild anaemia with a haemoglobin of $5.3 \mathrm{~g} \%$ normal transaminases, both elevated LDH and $\mathrm{C}$ reactive protein, total bilirubin of $1.2 \mathrm{mg} / \mathrm{dl}$, and significantly high blood glucose level. Coagulation parameters showed a Prothrombin
Time of 11 seconds, Activated Partial Thromboplastin Time of 39.8 seconds and International Normalized Ratio of 1.72. The serum lipase was within normal levels. Also both x-rays of the abdomen and thorax were normal. Abdominal ultrasound was inconclusive. Then, a CT of the abdomen showed a focal lesion in the 3rd hepatic segment of the left lobe suggestive of inflammatory lesion like abscess (Figure 1) without clear signs of pancreatic damage. The MRI (Figure 2) of the abdomen confirmed the presence of an oval image on the left hepatic

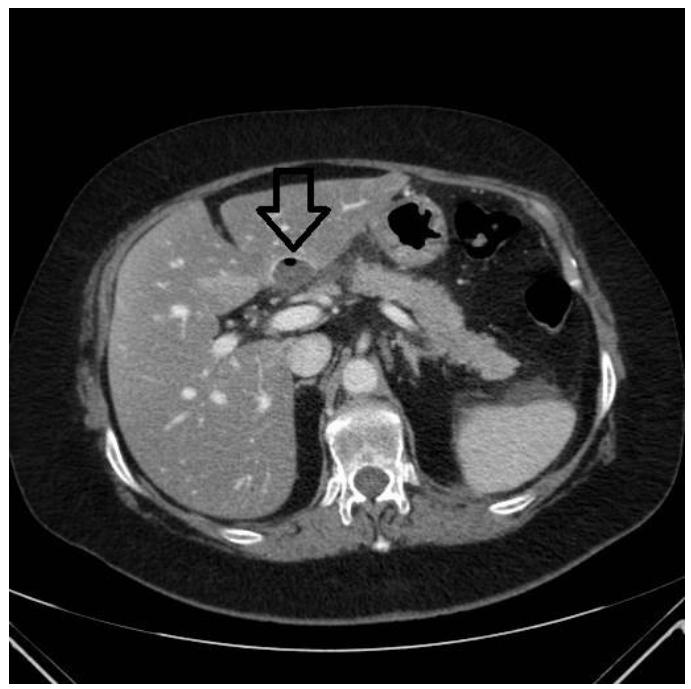

Figure 1. Computed Tomography of the abdomen showed a subcapsular intrahepatic lesion like abscess.

Correspondence to: Massimo Bolognesi, Internal General Medicine, Department of General Practice Medicine, AUSL della Romagna-District of Cesena, Via Ungaretti 49447521 Cesena, Italy, Tel: +390547645074; E-mail: massbolo1@tin.it

Key words: acute epigastric pain, intrahepatic focal lesion, computed tomography, magnetic resonance

Received: September 08, 2016; Accepted: October 19, 2016; Published: October 22,2016 


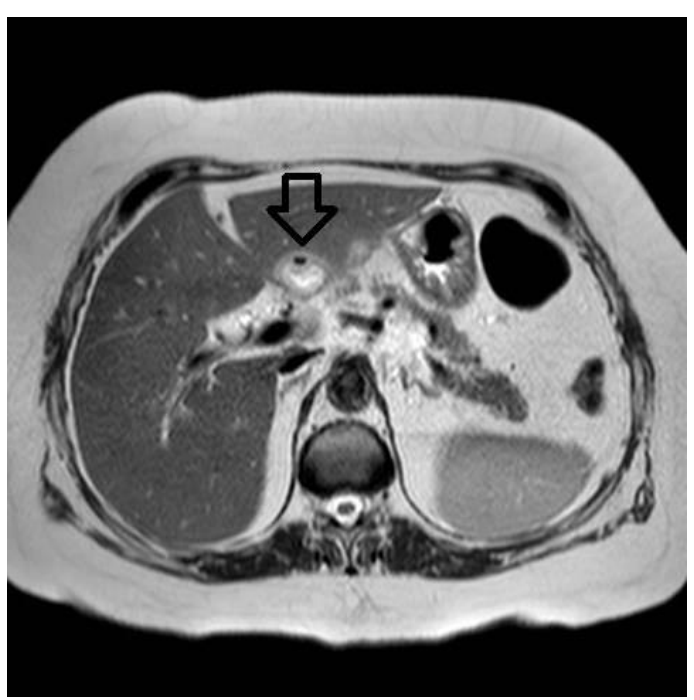

Figure 2. Magnetic Resonance of the abdomen showed the same intrahepatic oval lesion with fluid collection.

lobe with features of inflammatory lesion. In order to detect its origin and particularly the source of suspected infection an echocardiography was performed resulting negative for vegetations by endocarditis. Also the endoscopic examinations of the digestive tract (i.e., a gastroscopy and colonoscopy), blood cultures, coproculture and parasitological examination of feces were normal. The patient was treated in a conservative way with broad-spectrum antibiotics (piperacillin/ tazobactam + ciprofloxacin) as indicated by infectious disease doctors with normalization of inflammatory markers (CRP discharge $2.7 \mathrm{WBC}$ $6820)$. Finally, she was discharged with the diagnosis of "sepsis due to hepatic abscess".

\section{Discussion}

Subcapsular fluid collections around or inside the liver may arise from a variety of sources. An understanding of the anatomy of the fascial structures is important when interpreting imaging studies, such as computed tomography (CT), and correctly diagnosing such fluid collections [1]. Liver abscesses may extend into the subcapsular space from other sources [2]. Less commonly, fluid may accumulate from a ventriculoperitoneal shunt if the tube migrates out of place [3], or during an episode of acute pancreatitis [4]. Hepatic abscesses can be classified as pyogenic, amebic, or fungal [5]. Pyogenic or bacterial abscess may be caused by several factors and is classified by the route of entry of the organisms. Infections may arise from the biliary tract, portal vein and hepatic artery or by direct extension [6]. Infections arising from the biliary tract are the most common and result in $30 \%$ to $50 \%$ of the total number of pyogenic abscesses. The resultant cholangitis leads to liver abscesses, frequently multiple. The infecting organism varies according to the site of entry. In biliary or portal vein sepsis, the organisms are enteric and usually polymicrobial. Staphylococcus aureus is evident in $20 \%$ of cases and is confirmed predominantly by haematogenous spread [6]. Acute pancreatitis is defined as an acute inflammatory process of the pancreas with variable involvement of other regional tissues or remote organ systems [7] It is further classified into mild and severe forms. The Former is associated with minimal organ dysfunction and uneventful recovery, and the Latter with pancreatic necrosis which may lead to organ failure and/or local complications [8-11]. Hereditary angioedema (HAE) is an infrequent disorder which may be a potential cause of acute pancreatitis [12,13]. Pancreatic pseudocysts are a well recognized and common complication of acute and chronic pancreatitis. It is estimated that $20 \%$ of pseudocysts are extrapancreatic [14]. Although pancreatic pseudocysts can form anywhere in the abdomen, intrahepatic occurrence is a rare but always possible event [15]. They occur predominantly in the left lobe of the liver as a result of extension of fluid from the lesser sac into the leaves of the hepatogastric ligament [16]. Clinical symptoms are usually related to the underlying inflammatory pancreatic disease. Elevated serum and urinary amylase levels should arouse suspicion for this condition [16], however some literature reports cases of acute pancreatitis without the increase of pancreatic enzymes [17]. A correct diagnosis is not difficult with imaging when other signs of acute pancreatitis are present, but equivocal diagnosis may happen if pancreatic damage in the imaging is underlying [18]. At Computed Tomography of the abdomen, a mature intrahepatic pseudocyst can appear as a well-defined, subcapsular, homogeneous, hypoattenuating mass surrounded by a thin fibrous capsule like abscess [15]. Literature also reports that acute pancreatitis may be complicated by liver abscess [19]. Cross-sectional imaging is a reliable tool for diagnosing hepatic abscesses in the appropriate clinical setting, but because of nonspecific radiological features percutaneous biopsy may be required for a definitive diagnosis in patients with equivocal clinical symptoms. [20,21]. The key points of this case report are the following: 1 ) the presentation of disease with symptoms of acute onset of epigastric pain radiating to the left shoulder in the absence of gastric perforation or other gastrointestinal disease; 2) The history of idiopathic angioedema in a patient requiring the administration of intramuscular steroid therapy; 3) the hospital diagnosis of sepsis due to hepatic abscess; 4) the lack of the mandatory percutaneous procedure by biopsy and drainage of intrahepatic lesion that could have produced a correct diagnosis. The author is quite sure that the presentation and evolution of this case was suggestive for acute pancreatitis complicated by intrahepatic fluid collection as reported in many cases in the literature. For a correct diagnosis it would have been necessary to perform a percutaneous needle aspiration and drainage of the collections of intra-hepatic lesion, to examine the fluid through the exams of pancreatic enzymes. Especially if the lesion was suspected to be a liver abscess with sepsis in progress. To diagnose sepsis or systemic inflammatory response syndrome (SIRS) at least two of the following clinical criteria must be present: 1) Temperature $>38^{\circ} \mathrm{C}$ or $<36^{\circ} \mathrm{C}$; 2) Heart rate $>90 / \mathrm{min}$; 3) Respiratory rate $>20 /$ min or $\mathrm{PaCO}_{2}<32 \mathrm{~mm}$ $\mathrm{Hg}(4.3 \mathrm{kPa})$; 4) White blood cell count $>12000 / \mathrm{mm}^{3}$ or $<4000 / \mathrm{mm}^{3}$ or $>10 \%$ immature bands [22]. In this case report only one of these criteria was present. The current practice in the medical field is to rule out pancreatitis if the serum lipase levels are normal, but the current case may show that patients can have mild pancreatitis in absence of elevated amylase and/or lipase levels. The aim of this case report is to confirm that while uncommon, acute pancreatitis with normal serum amylase and lipase is a phenomenon which can occur, and rarely associated to normal pancreatic imaging. Therefore, the evidence by imaging of intrahepatic lesion with characteristic of fluid collections may be a intrahepatic localization of pancreatic pseudocyst or less likely an hepatic abscess. This suggests that further research is needed to better understand the mechanisms underlying the development of acute pancreatitis and the role that these digestive enzymes play. Hence the suspicion of pancreatitis should be high in patients with classic signs and symptoms.

\section{Conclusion}

Finally, in what way does this case report contribute to expand our current knowledge and improve clinical practice? Well, physician 
should keep in mind that intrahepatic pseudocyst or hepatic abscess are two conditions which can happen when an intrahepatic lesion with fluid collection is found in patients with features of recent episode of acute epigastric pain that suggested acute pancreatitis, even though imaging or laboratory analysis are unclear or equivocal. Tomographic scannings and high level of amylase in the collection play an important role in diagnosing this complication. In conclusion an early management perspective with percutaneous drainage of intrahepatic lesions of undetermined nature, such as pseudocysts or abscess, must be resorted to in the face of rapidly growing collections.

\section{Acknowledgements}

The author thanks her patient, who gave permission to publish this case.

\section{Conflict of interest}

The author declares to have no conflicts of interest.

\section{References}

1. Korobkin M, Silverman PM, Quint LE, Francis IR (1992) CT of the extraperitoneal space: normal anatomy and fluid collections. AJR Am J Roentgenol 159: 933-942. [Crossref]

2. Lee CH, Leu HS, Wu TS, Su LH, Liu JW (2005) Risk factors for spontaneous rupture of liver abscess caused by Klebsiella pneumoniae. Diagn Microbiol Infect Dis 52: 7984. [Crossref]

3. Reddy SC (1987) Subcapsular hepatic abscess: a rare complication of ventriculoperitoneal shunt. South Med J 80: 1309-1311. [Crossref]

4. Mofredj A, Cadranel JF, Dautreaux M, Kazerouni F, Hadj-Nacer K, et al. (2000) Pancreatic pseudocyst located in the liver: a case report and literature review. $J$ Clin Gastroenterol 30: 81-83. [Crossref]

5. Mergo PJ, Ros PR (1997) MR imaging of inflammatory disease of the liver. Magn Reson Imaging Clin N Am 5: 367-376. [Crossref]

6. Liver Diseases: MedlinePlus. www.nlm.nih.gov. Retrieved 2015-06-20.

7. Bradley EL (1993) A clinically based classification system for acute pancreatitis.
Summary of the International Symposium on Acute Pancreatitis, Atlanta, Ga, September 11 through 13, 1992. Arch Surg 128: 586-590. [Crossref]

8. Safrit HD1, Rice RP (1989) Gastrointestinal complications of pancreatitis. Radiol Clin North Am 27: 73-79. [Crossref]

9. Siegel MJ, Sivit CJ (1997) Pancreatic emergencies. Radiol Clin North Am 35: 815-830, 814. [Crossref]

10. Vujic I (1989) Vascular complications of pancreatitis. Radiol Clin North Am 27: 81-91. [Crossref]

11. Sureka B, Bansal K, Patidar Y, Arora A (2016) Imaging lexicon for acute pancreatitis 2012 Atlanta Classification revisited. Gastroenterol Rep (Oxf) 4: 16-23. [Crossref]

12. Damir Matesic, Evans R Fernández Pérez, Nicholas E Vlahakis, John B Hagan (2006) Acute pancreatitis due to hereditary angioedema. Annals of Allergy, Asthma \& Immunology 97: 611-614.

13. Cancian M, Vettore G, Realdi G (2011) An Uncommon Cause of Acute Pancreatitis Hereditary Angioedema-Induced Acute Pancreatitis Gastroenterology 140: 330-370

14. Hamm B, Franzen N (1993) Atypically located pancreatic pseudocysts in the liver, spleen, stomach wall and mediastinum: their CT diagnosis. Rofo 159: 522-527.

15. Murphy BJ, Casillas J, Ros PR, Morillo G, Albores-Saavedra J, et al. (1989) The CT appearance of cystic masses of the liver. Radiographics 9: 307-322. [Crossref]

16. Okuda K, Sugita S, Tsukada E, Sakuma Y, Ohkubo K (1991) Pancreatic pseudocyst in the left hepatic lobe: a report of two cases. Hepatology 13: 359-363. [Crossref]

17. Shah AM, Eddi R, Kothari ST, Maksoud C, DiGiacomo WS, et al. (2010) Acute pancreatitis with normal serum lipase: a case series. JOP 11: 369-372. [Crossref]

18. Koo BC, Chinogureyi A, Shaw AS (2010) Imaging acute pancreatitis. Br J Radiol 83: 104-112. [Crossref]

19. Kumar S, John F, Yegneswaran B (2016) A case of recurrent pancreatitis complicated by liver abscess. Journal of Gastroenterology, Pancreatology \& Liver Disorders 3:1.2

20. Mathieu D, Vasile N, Fagniez PL, Segui S, Grably D, et al. (1985) Dynamic CT features of hepatic abscesses. Radiology 154: 749-752.

21. Mortele KJ, Segatto E, Ros PR (2004) The infected liver: radiologic-pathologic correlation. Radiographics 24: 937-955.

22. Bone RC, Balk RA, Cerra FB (1992) American College of Chest Physicians/Society of Critical Care Medicine Consensus Conference: definitions for sepsis and organ failure and guidelines for the use of innovative therapies in sepsis. Crit Care Med 20: 864-874.

Copyright: (2016 Bolognesi M. This is an open-access article distributed under the terms of the Creative Commons Attribution License, which permits unrestricted use, distribution, and reproduction in any medium, provided the original author and source are credited. 